\title{
Development of a neutral diketopyrrolopyrrole phosphine oxide for the selective bioimaging of mitochondria at the nanomolar
}

\section{level}

\author{
Thais F. Abelha, ${ }^{a}$ Graeme Morris, ${ }^{b}$ Sandro M. Lima, ${ }^{c}$ Luis H. C. Andrade, ${ }^{c}$ Andrew J. McLean, ${ }^{b}$ \\ Cameron Alexander, ${ }^{a}$ Jesus Calvo-Castro*d and Callum J. McHugh ${ }^{* b}$ \\ Abstract: Development of novel bioimaging materials that exhibit

\section{Introduction} \begin{abstract}
organelle specific accumulation continues to be at the forefront of research interests and efforts. Among the various subcellular organelles, mitochondria, which are found in the cytoplasm of eukaryotic cells, are of particular interest in relation to their vital function. To date, most molecular probes that target mitochondria utilise delocalised lipophilic cations such as triphenylphosphonium and pyridinium. However, the use of such charged motifs is known to be detrimental to the working function of the mitochondrial transmembrane potential and there remains a strong case for development of neutral mitochondrial fluorescent probes. Herein, we demonstrate for the first time the exploitation of diketopyrrolopyrrolebased chemistries for the realisation of a neutral fluorescent probe that exhibits organelle specific accumulation within the mitochondria at the nanomolar level. The synthesised probe, which bears a neutral triphenylphosphine oxide moiety, exhibits a large Stokes shift and high fluorescence quantum yield in water, both highly sought-after properties in the development of bioimaging agents. In-vitro studies reveal no interference with cell metabolism when tested for the human MCF7 breast cancer cell and nanomolar subcellular organelle colocalisation with commercially available mitochondrial staining agent Mitotracker Red. In light of its novelty, neutral structure and the preferential accumulation at nanomolar concentrations we anticipate this work to be of significant interest for the increasingly larger community devoted to the realisation of neutral mitochondrial selective systems and more widely to those engaged in the rational development of superior organic architectures in the biological field.
\end{abstract}

[a] Dr T. F. Abelha, Prof. C. Alexander School of Pharmacy The University of Nottingham University Park NG72RD Nottingham, UK.

[b] Dr G. Morris, Dr A. J. McLean, Dr C. J. McHugh School of Computing, Engineering and Physical Sciences University of the West of Scotland

PA12BE

Paisley, UK.

E-mail: callum.mchugh@uws.ac.uk

[c] Dr S. M. Lima, Dr L. H. C. Andrade Grupo de Espectroscopia Óptica e Fototérmica Universidade Estadual de Mato Grosso do Sul CP 351, 79804-970

Dourados, MS, Brazil.

[d] Dr J. Calvo-Castro

School of Life and Medical Sciences

University of Hertfordshire

AL109AB

Hatfield, UK.

E-mail: j.calvo-castro@herts.ac.uk

Supporting information for this article is given via a link at the end of the document.
Over the last two decades there has been increasing interest in the rational development of novel bioimaging materials which exhibit organelle specific accumulation..$^{[1-8]}$ Among those subcellular organelles of interest, mitochondria, which are found in the cytoplasm of eukaryotic cells, are of particular note and significant efforts have been devoted towards the realisation of novel staining agents. ${ }^{[5-8]}$ Mitochondria denote the principal energy-producing compartment in most eukaryotes. Their function is critical in a number of vital process such as adenosine triphosphate (ATP) production, central metabolism and apoptosis, ${ }^{[9,10]}$ and irregularities in these functions are intimately linked to degenerative diseases such as Alzheimer's and Parkinson's as well as cardiac complications and cancer. ${ }^{[6-10]}$ Currently, a range of diagnostic imaging techniques, such as radioisotope labelling, magnetic resonance imaging and positron emission tomography, are employed for monitoring subcellular organelles. ${ }^{[6,11]}$ Recently, approaches exploiting fluorescence emission have been developed for the investigation of specific organelles such as mitochondria, representing a powerful class of imaging strategies, on account of their ability to translate molecular recognition into highly discriminative and easily detected optical signals. ${ }^{[6,12,13]}$

The mitochondrion, which is structurally characterised by a double membrane separated by the intermembrane space, exhibits a highly negative transmembrane potential as a result of the process of mitochondrial respiration whereby the proton pumps in the inner mitochondrial membrane transport protons onto the intermembrane space. ${ }^{[11,14-17]}$ This large potential distinguishes it from other subcellular organelles and has inspired the successful development of optically active probes that exploit it selectively. Thus, systems containing delocalised lipophilic cations (DLCs) such as triphenylphosphonium or pyridinium cation, which are present in the majority of mitochondria targeting probes to date and which are believed to improve membrane transport and increase mitochondria probe accumulation, have been widely used to ensure selectivity towards this subcellular compartment attracted by the negative potential of the membrane, whilst maintaining an otherwise non-polar framework in most cases. ${ }^{[6-8,18]}$ In this regard, a number of organic dyes such as fluorescein, ${ }^{[19,20]}$ coumarin $^{[21,22]}$ and rhodamine ${ }^{[23,24]}$ have been utilised, as the fluorescent tag to which DLCs are anchored. However, their biological applications are often limited by their photophysical and/or biological properties. Importantly, and despite the large efforts devoted to the development of charged fluorescent probes as selective staining agents for mitochondria, the known negative effects of cationic systems are often overlooked. In short, these lipophilic cationic structures carry the risk of reducing the mitochondria membrane potential (MMP), 
which is associated to detrimental effects in their critical working function. Further, probe leakage through the membrane can also be attributed to a reduction in the MMP, hence decreasing the efficiency of the staining agents. ${ }^{[7,18]}$ In response to the latter, the development of neutral fluorescent probes that maintain selective targeting of mitochondria and alleviate those concerns would represent major innovations and is therefore highly warranted. ${ }^{[25]}$ Whilst access to the mitochondria intermembrane space is relatively straightforward, facilitated by means of large proteinbased pores in the outer membrane, the inner membrane is characterised by a much more restricted permeability, loaded with proteins that play a role in electron transport and ATP synthesis processes. Transport across and onto the mitochondrial matrix can only occur aided by specific membrane transport proteins. ${ }^{[14,18]}$ The latter has motivated the development of peptide-based mitochondrion staining agents whereby specific protein-ligand type interactions are favoured, which could foster the rational development of novel non-peptidic alternatives for mitochondrial staining species. ${ }^{[8,18]}$

In response to these findings and motivated by the latter, we are engaged in the development of novel non-charged systems that can exhibit selective organelle accumulation exploiting diketopyrrolopyrrole (DPP) chemistries. DPPs were first employed in industry as high performance pigments on account of their desirable properties such as brightness as well as light and weather fastness and then widely used as charge transfer mediating materials in optoelectronic applications. ${ }^{[26-30]}$ Despite the promising performance of some DPP-based materials in the bioimaging field, their utilisation as mitochondria specific staining agents is still underexploited and fully relies on the use of charged moieties. ${ }^{[31-35]}$ In addition, the realisation of DPP-based staining agents at the nanomolar level still represents a challenge in the field. Herein, we report the rational development, in-depth photophysical analysis and in-vitro imaging of a novel neutral DPP-based selective mitochondrial staining agent (PhODPP) at the nanomolar level. To design the probe, we initially explored the ability of a DPP material bearing a non-charged triphenylphosphine group (PhDPP) to exhibit preferential subcellular organelle accumulation for mitochondria, based on previous reports exploiting triphenylphosphonium cation. We observed that whilst cell viability studies for PhDPP were consistent with no interference of the probe with the cell metabolism, it exhibited relatively low cell uptake at commonly employed concentrations, which could be partially ascribed to its low fluorescence quantum yield and accumulation in subcellular organelles other than mitochondria. Employing in-silico design, we formulated a superior alternative whereby chemical oxidation of the phosphine to phosphine oxide precludes thermodynamically favoured photoinduced electron transfer (PET) exhibited by PhDPP, resulting in a remarkable 7-fold increase in the fluorescence quantum yield in water. Importantly, the oxidised probe was also characterised by no interference with cell metabolism when tested for the human MCF7 breast cancer cell and to exhibit larger cell uptake and nanomolar organelle accumulation within the mitochondria, which, in the absence of a charged motif, we attribute to a reversal of the non-covalent interactions of the triphenylphosphine oxide unit with protein binding pockets within the inner mitochondrial membrane. As such, our oxidised probe denotes the first non-charged DPP based system for selective imaging of this subcellular organelle reported to date. In addition, it represents the first DPP-based bioimaging probe with sensitivity at the nanomolar level and comparable performance to the commercially available Mitotracker Red staining agent. The latter, associated to its neutral characteristics makes this probe a major innovation in the field.

\section{Results and Discussion}

\section{Synthesis, photophysical properties and subcellular accumulation of PhDPP}

Inspired by the large number of reported probes exhibiting selective mitochondria accumulation by exploiting the triphenylphosphonium moiety and our desire to develop noncharged fluorophores that exhibit preferential subcellular accumulation for this organelle, we initially synthesised an asymmetric model DPP bearing a non-cationic triphenylphosphine unit anchored to one of the core phenyl rings (Scheme 1). Although most efforts devoted to the realisation of mitochondrial staining agents are based around the utilisation of charged moieties such as triphenylphosphonium or pyridinium cations, we wanted to explore whether a neutral triphenylphosphine substitution would facilitate probe accumulation within the mitochondria. Synthesis of the model DPP phosphine derivative, PhDPP was carried out via an optimised palladium catalysed phosphination ${ }^{[36]}$ of a monoiodinated DPP ${ }^{[37]}$ using $\mathrm{Pd}(\mathrm{OAc})_{2}$ as shown in Scheme 1 (SI.1 ESIt). Phosphination of the mono-brominated DPP or the use of $\mathrm{Pd}\left(\mathrm{PPh}_{3}\right)_{4}$ as catalyst gave lower yields of the target compound. Alternatively, conventional organolithium and Grignard mediated phosphination reactions of the mono-iodinated and monobrominated DPP were explored, however neither approach gave any conversion to the corresponding phosphine derivative.

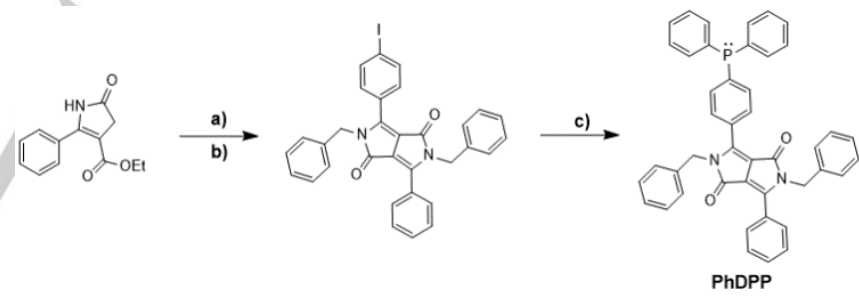

Scheme 1. Synthesis of PhDPP. a) 4-iodobenzonitrile, Na, t-amyl alcohol, reflux; b) $\mathrm{BnBr}$, DMF, K2CO3, $120^{\circ} \mathrm{C}$; c) $\mathrm{PPh} 3, \mathrm{Pd}(\mathrm{OAc}) 2, \mathrm{DMF}, 120^{\circ} \mathrm{C}$.

The absorption and fluorescence emission properties of PhDPP were experimentally determined in solution in the solvents of acetonitrile, dichloromethane, dimethyl sulfoxide (DMSO) and water $(0.2 \%$ DMSO as co-solvent). Figure 1 illustrates the normalised absorption and fluorescence emission spectra for this probe in the investigated solvents. In all cases, absorption spectra for the probe were broad, displaying no vibronic structure and a small bathochromic shift on progression to water. Fluorescence emission spectra displayed vibronic progression and spectral shape irrespective of the excitation wavelength (SI.2 ESIT), with the intensity of the $0-0$ band being greater than that of the $0-1$ band in all cases but water, thus consistent with relatively small structural changes on progression to excited state geometries. In turn, the fluorescence emission spectrum of PhDPP in water illustrates a reversal of the relative intensities of the first and second vibrational bands and an associated bathochromic shift 
( $\lambda_{\mathrm{em}}=549$ and $604 \mathrm{~nm}$ in DMSO and water, respectively) and larger Stokes shift when compared to the spectra in other solvents $\left(\Delta \lambda_{\max }=2749\right.$ and $4233 \mathrm{~cm}^{-1}$ in DMSO and water, respectively). The latter denotes a highly desirable property as to diminish the probability of self-absorption that could compromise the potential application of the probe as a bioimaging agent.

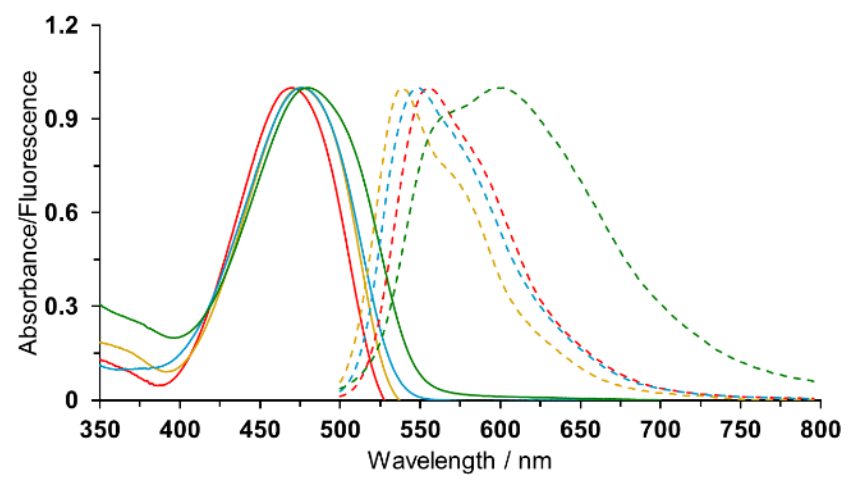

Figure 1. Normalised absorption (solid line) and fluorescence emission (dashed line) spectra of PhDPP in acetonitrile (red), dichloromethane (yellow), DMSO (blue) and water (0.2\% DMSO, green).

Underpinned by a detailed understanding of the photophysical properties of PhDPP (see Table 1 for details), we sought to test its cell viability and ability to exhibit preferential subcellular organelle accumulation. Firstly, the cell viability of the triphenylphosphine bearing DPP was determined for the human cell MCF7 breast cancer cells by the resazurin based metabolic assay PrestoBlue. Cells were treated with PhDPP at concentrations ranging from $0.2-21.4 \mu \mathrm{g} / \mathrm{mL}(0.31-33 \mu \mathrm{M})$ and incubated for $1 \mathrm{~h}$. In all cases we observed viabilities higher than $90 \%$, thus indicating no interference with the cell metabolism (Figure 6A). Subsequently, we went on to explore the intracellular uptake and localisation of PhDPP. We observed that at a typical concentration previously utilised for bioimaging probes $(1 \mu \mathrm{g} / \mathrm{mL}=$ $1.5 \mu \mathrm{M}$ ), the cellular uptake is relatively low (SI.3 ESIT). Colocalisation of the developed probe with commercially available mitochondrial staining agent Mitotracker Red CMXRos (250 nM), revealed that PhDPP does not preferentially accumulate within the mitochondria (SI.3 ESIT). Via analysis of the photophysical properties of PhDPP and the subcellular localisation data, we ascribe the aforementioned findings to: i) the inability of the neutral triphenylphosphine moiety in fostering strong binding interactions with appropriate proteins within the inner mitochondrial membrane, and ii) to a lesser extent the low fluorescence quantum yield $\left(\Phi_{f}=0.08 \pm 0.02\right.$ in water) that could compromise bioimaging irrespective of subcellular organelle accumulation.

Rational development, synthesis and photophysical properties of PhODPP

Next, we engaged in the rational development of a potentially superior, whilst structurally related, probe whereby fluorescence efficiency as well as cell uptake and mitochondrial accumulation could be improved. First, we proposed via analysis of PhDPP structure, that the low experimentally determined fluorescence quantum yield could occur as a result of a thermodynamically favoured intramolecular PET from the lone pair of electrons in the phosphine within the triphenylphosphine moiety onto the DPP core, hence quenching the inherently high fluorescence efficiency characteristic of diketopyrrolopyrroles (Figure 2). ${ }^{[38,39]}$ Along those lines, we hypothesised that chemical oxidation of the triphenylphosphine moiety to its oxidised, triphenylphosphine oxide counterpart could preclude intramolecular PET and increase the fluorescence quantum yield of the probe. In addition, and importantly, this structural change would significantly reverse the type of non-covalent interactions in which the probe could engage with amino acids within the proteins of the inner mitochondrial membrane, hence potentially contributing to promoting preferential accumulation.

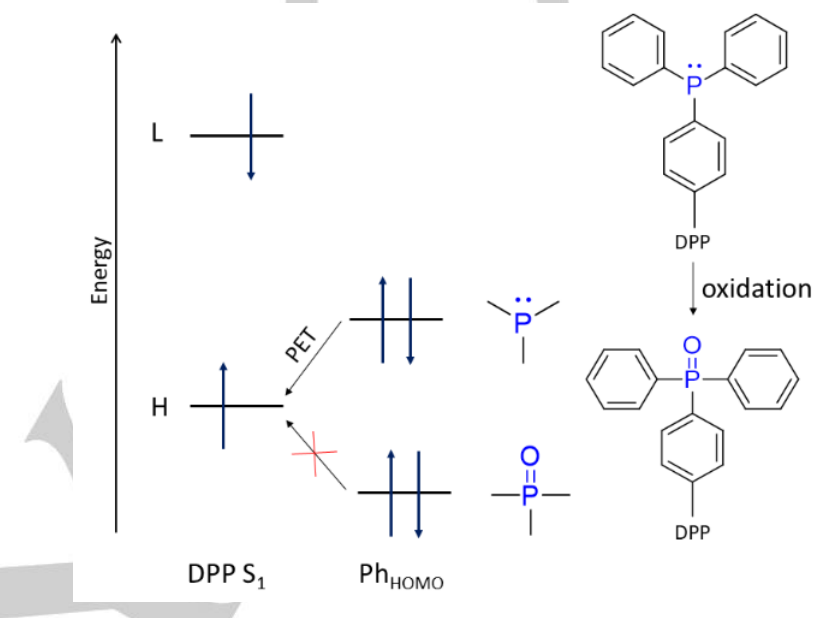

Figure 2. Illustration of the proposed intramolecular photoinduced electron transfer quenching mechanism in PhDPP and turn-on effect in PhODPP upon

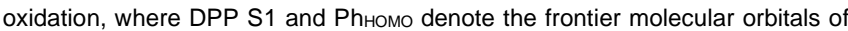
the DPP singlet excited state and HOMO energy levels of the phosphine/phosphine oxide moiety, respectively.

To test this hypothesis in-silico, we went on to optimise the geometries of PhDPP and its oxidised counterpart, PhODPP by means of quantum mechanical calculations employing Thrular's global-hybrid meta-GGA density functional M06-2X at the $6-31 \mathrm{G}^{*}$ level. In agreement with the illustration in Figure 2, we observed a large decrease in the HOMO energy of the triphenylphosphine/triphenylphosphine oxide moiety upon oxidation $\left(\mathrm{E}_{\text {HOMO(Ph) }}=-7.26\right.$ and $-8.47 \mathrm{eV}$ for the phosphine/phosphine oxide moiety in PhDPP and PhODPP respectively), which is consistent with our hypothesis that chemical oxidation of PhDPP would inhibit quenching and maximise fluorescence emission via a turn-on effect mechanism. Underpinned by these findings, PhODPP was synthesised following the previously described optimised method and could be readily separated from PhDPP via preparative chromatography. In all investigated solvents, both the absorption as well as fluorescence emission spectra of PhODPP exhibited negligible differences when compared to PhDPP spectra (Figure 1), which highlights that these properties are determined by the DPP moiety. As illustrated in Figure 3, the absorption spectra of PhODPP in all the investigated solvents were broad and displayed no vibronic structure. In turn, fluorescent emission spectral shape was irrespective of excitation wavelength (SI.2 ESI†) and exhibited vibronic progression, with the intensity of the $0-0$ band being greater than that of the $0-1$ band in all cases except water, where 
the opposite relative orientation was observed, resulting in a significant bathochromic shift for PhODPP in water when compared to the spectra in the other investigated solvents $\left(\lambda_{\mathrm{em}}=\right.$ 550 and $599 \mathrm{~nm}$ in DMSO and water, respectively).

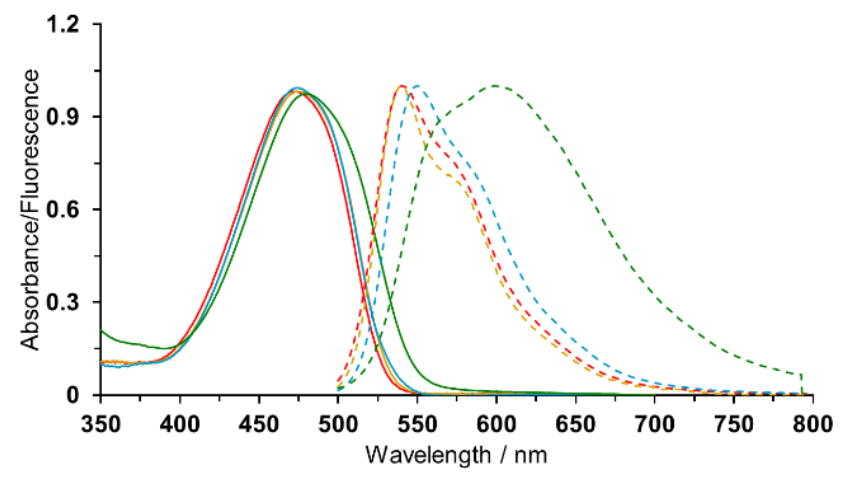

Figure 3. Normalised absorption (solid line) and fluorescence emission (dashed line) spectra of PhODPP in acetonitrile (red), dichloromethane (yellow), DMSO (blue) and water (0.2\% DMSO, green)

In short, the latter can be ascribed to larger solvent induced planarity of the geometry of the relaxed first singlet excited state with respect to the optimised ground state, hence resulting in a greater relative displacement of the respective potential energy surfaces along the nuclear coordinates axis and the observed reversal of the relative intensity of the first two vibrational bands. Along these lines, similarly to the case of PhDPP, we report significant Stokes shift also for the oxidised probe $\left(\Delta \lambda_{\max }=2871\right.$ and $4096 \mathrm{~cm}^{-1}$ for PhODPP in DMSO and water, respectively), which, again, minimises the probability of self-quenching, denoting a highly sought-after property in the realisation of novel fluorescent probes for bioimaging applications.

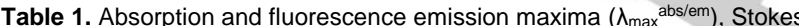
shifts $\left(\Delta \lambda_{\max }\right)$, fluorescence lifetimes $\left(\mathrm{T}_{\mathrm{f}}\right)$ and photoluminescence quantum yields $\left(\Phi_{\mathrm{f}}\right)$ of PhDPP and PhODPP in acetonitrile $(\mathrm{MeCN})$, dichloromethane (DCM), dimethyl sulfoxide (DMSO) and water $\left(\mathrm{H}_{2} \mathrm{O}\right)$.

\begin{tabular}{|c|c|c|c|c|}
\hline PhDPP & $\lambda_{\max }{ }^{\mathrm{abs} / \mathrm{em}} / \mathrm{nm}$ & $\Delta \Lambda_{\max } / \mathbf{c m}^{-1}$ & Tf / ns & $\Phi f$ \\
\hline $\mathrm{MeCN}$ & $471 / 556$ & 3246 & 6.21 & $0.20 \pm 0.04$ \\
\hline DCM & $476 / 539$ & 2456 & 5.59 & $0.25 \pm 0.05$ \\
\hline DMSO & $477 / 549$ & 2749 & 5.87 & $0.23 \pm 0.06$ \\
\hline $\mathrm{H}_{2} \mathrm{O}^{\text {[a] }}$ & $481 / 604$ & 4234 & 2.33 & $0.08 \pm 0.02$ \\
\hline PhODPP & $\lambda_{\max }{ }^{\mathrm{abs} / \mathrm{em}} / \mathrm{nm}$ & $\Delta \lambda_{\max } / \mathbf{c m}^{-1}$ & tf / ns & Фf \\
\hline $\mathrm{MeCN}$ & $471 / 540$ & 2713 & 6.38 & $0.88 \pm 0.05$ \\
\hline DCM & $474 / 539$ & 2544 & 6.24 & $>0.93$ \\
\hline DMSO & $475 / 550$ & 2871 & 6.38 & $>0.95$ \\
\hline $\mathrm{H}_{2} \mathrm{O}^{[\mathrm{a}]}$ & $481 / 599$ & 4096 & 3.62 & $0.56 \pm 0.05$ \\
\hline
\end{tabular}

[a] $0.2 \%$ DMSO.
Fluorescence lifetimes and quantum yields were also determined in all investigated solvents for PhODPP as well as for its nonoxidised counterpart, PhDPP for comparison. Fluorescence lifetimes were observed to be comparable for both probes in the same solvent (e.g. $T_{f}=6.21$ and $6.38 \mathrm{~ns}$ for PhDPP and PhODPP in acetonitrile, respectively) and to furthermore be lower in water ( $\mathrm{T}_{\mathrm{f}}=2.33$ and $3.62 \mathrm{~ns}$ for PhDPP and PhODPP, respectively). In contrast, and in line with our initial hypothesis and quantum mechanical calculations, a dramatic increase in fluorescence quantum yield was observed upon chemical oxidation (e.g. $\Phi_{\mathrm{f}}=$ $0.20 \pm 0.04$ and $0.88 \pm 0.05$ for PhDPP and PhODPP in acetonitrile, respectively). Remarkably, we report a 7 -fold increase observed in fluorescence quantum yield in water on going from PhDPP to its oxidised counterpart $\left(\Phi_{f}=0.08 \pm 0.02\right.$ and $0.56 \pm 0.05$ for PhDPP and PhODPP, respectively), hence making the oxidised probe a potent fluorophore even in water. The even lower fluorescence quantum yield in water when compared to other investigated solvents for PhDPP, could be attributed to a more efficient PET in polar solvents.

Motivated by these findings, we subsequently investigated the cellular uptake and organelle accumulation of PhODPP, with an aim to experimentally determine whether the structural changes would also result in preferential probe bioaccumulation within the mitochondria.

Cell viability, cellular uptake and subcellular localisation of PhODPP

Similarly to the case of PhDPP, we determined the cell viability of PhODPP for the human MCF7 breast cancer cells by the resazurin based metabolic assay PrestoBlue by treating the cells with PhODPP at concentrations ranging from 0.2 to $21.4 \mu \mathrm{g} / \mathrm{mL}$ $(0.30-32 \mu \mathrm{M})$ and incubation for $1 \mathrm{~h}$. We report observed viabilities higher than $90 \%$ in all cases, consistent with no interference of the probe with the cell metabolism (Figure 4A).

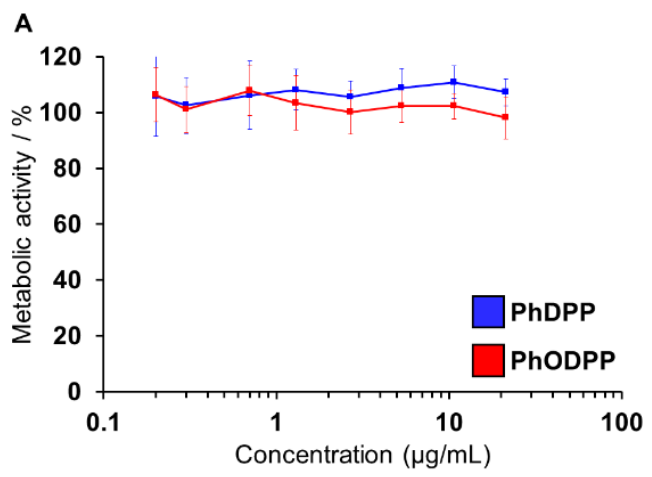

B

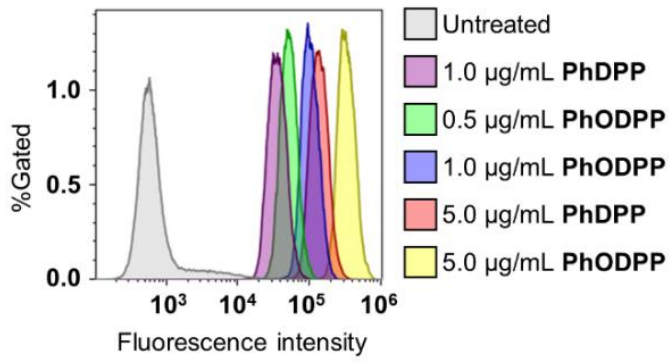

Figure 4. A MCF7 cell viability $1 \mathrm{~h}$ incubation with serial dilutions $(0.2-21.4$ $\mu \mathrm{g} / \mathrm{mL}$ ) of PhDPP (blue) and PhODPP (red), $(\mathrm{n}=3)$. B Flow cytometry histogram of human MCF7 breast cancer cells treated with different concentrations of PhDPP and PhODPP. $\lambda_{\mathrm{exc}}=488 \mathrm{~nm}$ and $\lambda_{\mathrm{em}}=514-542 \mathrm{~nm}$ 
Next, we investigated the intracellular uptake and subcellular organelle localisation of PhODPP and compare these to the results observed for the non-oxidised counterpart, PhDPP. We report a significantly higher cell uptake for PhODPP when compared to PhDPP, as illustrated in Figure 4B. The latter can be ascribed to the observed increase in fluorescence emission efficiency (vide supra) but also to a better accumulation within the mitochondria as a result of the structural differences between triphenylphosphine and triphenylphosphine oxide. Importantly and contrary to the findings observed for PhDPP, PhODPP presented preferred co-localised organelle accumulation with the commercial mitochondria staining agent Mitotracker Red. Therefore, our oxidised probe, PhODPP, represents, to the best of our knowledge, the first report of a neutral DPP-based staining agent with preferential mitochondrial localisation and highlights the relevance that small chemical modifications can bear on subcellular organelle accumulation. In addition, the non-charged character of the developed probe would further alleviate common shortcomings in relation to probe-induced changes to the MMP, which denotes a critical point of concern in the development of novel systems for mitochondria imaging.

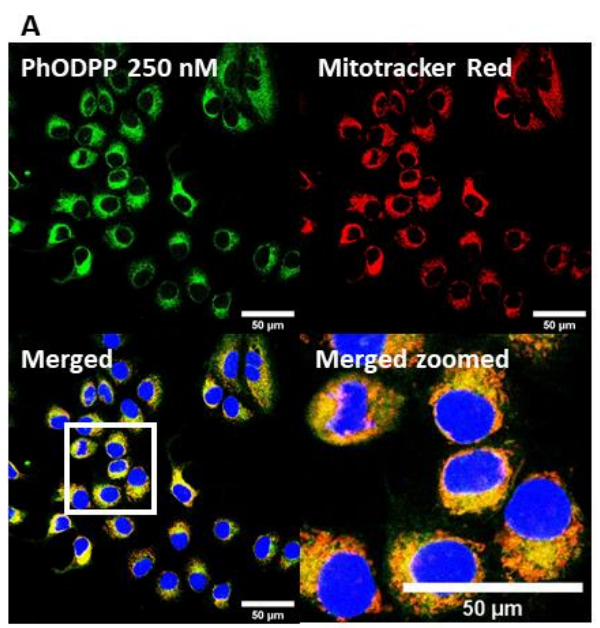

B

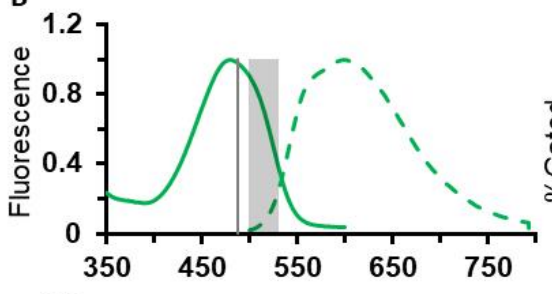

C
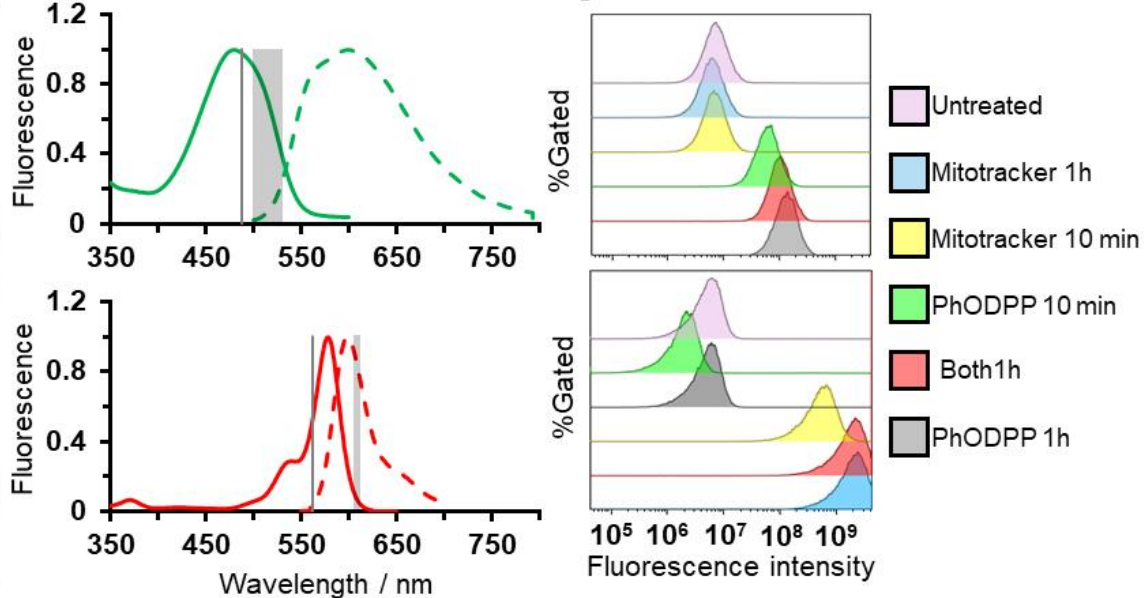

Figure 5. A MCF7 intracellular distribution of PhODPP $(250 \mathrm{nM}, 0.163 \mu \mathrm{g} / \mathrm{mL})$ and Mitotracker Red $(250 \mathrm{nM}, 0.133 \mu \mathrm{g} / \mathrm{mL})$. Scale bar represents $50 \mu \mathrm{M}$. B Normalised absorption (dashed line) and fluorescence emission (solid line) spectra of PhODPP (top) and Mitotracker Red (bottom) in water (0.2\% DMSO). Grey solid lines denote the excitation wavelengths ( $\lambda_{\text {exc }}=488$ and $561 \mathrm{~nm}$ for PhODPP and Mitotracker Red respectively) and grey bands illustrate spectral regions for PhODPP ( $\lambda_{\mathrm{em}}=500-526$ and 614-620 $\mathrm{nm}$ for PhODPP and Mitotracker Red respectively). C Flow cytometry histogram of MCF7 cells treated with $250 \mathrm{nM}$ concentrations of PhODPP and Mitotracker Red with $\lambda_{\mathrm{exc}}=488 \mathrm{~nm}$, spectral region $\lambda_{\mathrm{em}}=500-526 \mathrm{~nm}$ (top) and $\lambda_{\mathrm{exc}}=561 \mathrm{~nm}$, spectral region $\lambda_{\mathrm{em}}=614-620 \mathrm{~nm}$ (bottom).

To further investigate and benchmark the use of PhODPP as a staining agent for mitochondria, we quantified its accumulation within this subcellular organelle and compared it to that of Mitotracker Red. Cell treatment was followed with $250 \mathrm{nM}$ dosing of each probe at $10 \mathrm{~min}$ and $1 \mathrm{~h}$ incubation times, as well as simultaneous $250 \mathrm{nM}$ of both probes at $1 \mathrm{~h}$ incubation. As illustrated in Figure 5 (A) and by means of flow cytometry (C), it was determined that PhODPP and Mitotrocker Red exhibit cumulative cell uptake with increasing exposure time and detectable levels after $10 \mathrm{~min}$ incubation in both cases (Figure $5 \mathrm{C})$. The working concentration of the developed system is at the nanomolar level and therefore lower than that required for other previously reported DPP-based bioimaging agents for mitochondria. ${ }^{[1-35]}$ In this regard, although upon initial analysis of the flow cytometry histograms (Figure $5 \mathrm{C}$ ) reveal greater intensity for Mitotracker Red dosing when compared to our PhODPP probe, after careful evaluation, our results are consistent with comparable performance. In short, we ascribe the latter to the different extent to which the monitored spectral regions overlap with the emission spectra in each case $(1.31$ and $7.17 \%$ for PhODPP and Mitotracker Red, respectively, Figure 5 B). Lastly, simultaneous dosing of the cells with both probes yielded a reduction of their fluorescence intensity signals, which was observed to be lower in the case of the commercial probe. Although a more in-depth investigation would be required, the larger decrease in the case of PhODPP could be attributed to an efficient resonance energy transfer process as a result of the large spectral overlap between the absorption spectrum of Mitotracker Red and the fluorescence emission spectrum of our synthesised probe.

In summary, PhODPP denotes the first neutral DPP probe to exhibit mitochondrial accumulation (and one of the very few to do so irrespective of the chemistries exploited), performing at the nanomolar level, with comparable performance to the commercially available mitochondrial staining agent Mitotracker Red. In addition, PhODPP stock solutions in DMSO can be stored at $4-8{ }^{\circ} \mathrm{C}$, whilst stock solution of Mitotracker Red must be stored at $-20^{\circ} \mathrm{C}$, with stability compromised by the freeze and thaw cycle. Notably, PhODPP can also withstand the use of common cell preservatives (4\% PFA), which is desirable for a variety of cell characterisation assays.

\section{Conclusions}

In conclusion, we report the design, synthesis, photophysical properties and comprehensive in-vitro characterisation of a novel diketopyrrolopyrrole phosphine oxide, denoting the first neutral DPP based system exhibiting preferred mitochondrial accumulation at the nanomolar level. Inspired by the ubiquitous 
employment of delocalised lipophilic cations such as triphenylphosphonium to target mitochondria, we investigated whether a non-charged triphenylphosphine moiety anchored to a known fluorophore such as diketopyrrolopyrrole would still exhibit preference for that subcellular organelle. We observed relatively low cellular uptake and no preferential accumulation within our target organelle, which we ascribe to the inability of the triphenylphosphine moiety to engage in binding interactions with proteins within the mitochondrial inner membrane and to a lesser extent to its low fluorescence quantum yield as a result of efficient intramolecular photoinduced electron transfer. Underpinned by insilico approaches, we went on to synthesise a triphenylphosphine oxide analogue of our initial probe. We observed in line with our hypothesis, that PhODPP is a potent fluorophore as a result of chemical oxidation which precludes thermodynamically favourable intramolecular electron transfer. Cell viability studies using the human MCF7 breast cancer cell revealed no interference with the cell metabolism, with the phosphine oxide exhibiting a higher cell uptake when compared to our initial phopshine based attempt. Further, we report that this system demonstrates subcellular organelle colocalization with commercially available mitochondrial staining agent, Mitotracker Red at nanomolar concentrations. This system denotes the first report of a neutral diketopyrrolopyrrole-based fluorescent probe to selectively target mitochondria, therefore addressing known detrimental effects to the mitochondrial intermembrane potential associated with the use of charged molecular probes. Importantly, it is also the first DPP system, irrespective of the chemistries employed, to do so at nanomolar concentrations. Consequently, we anticipate this work to be of great relevance to those engaged in the development of mitochondrial bioimaging agents and more widely to those devoted to the realisation of superior fluorescent probes for bioimaging.

\section{Experimental Section}

Chemicals, reagents and instrumentation. NMR spectroscopy was carried out using a Bruker AV3 $400 \mathrm{MHz}$ instrument. FTIR spectroscopy was carried out by attenuated total reflectance using a Perkin Elmer, Spectrum One FTIR Spectrometer, with Universal ATR Sampling Accessory. Elemental analysis of Carbon, Hydrogen and Nitrogen (CHN) was carried out using a Perkin Elmer 2400 series $2 \mathrm{CHN}$ analyser. Melting points were determined employing a Stuart SMP20 melting point apparatus. HPLC grade dichloromethane was purchased from FisherScientific and used as supplied. Spectroscopic grade dried-acetonitrile was purchased from Sigma-Aldrich and was distilled and stored over $3 \AA$ molecular sieves (Sigma-Aldrich), which were oven dried before repeating the distillation process. Spectroscopy grade DMSO was purchased from Acros Organics and used as received. Steady-state absorption spectrophotometry measurements were performed using a Perkin-Elmer Lambda 40 UV-Vis spectrophotometer, operated by the software UV WinLab v.2.80.03 supplied by the manufacturer. Measurements were carried out in standard $10 \mathrm{~mm}$ match pair quartz cuvettes purchased from Hellma-Analytics. Steady-state fluorescence emission measurements were carried out employing a Perkin-Elmer LS50b luminescence spectrometer, controlled by means of the software FL WinLab v.4.00.03 supplied by the manufacturer. The photomultiplier tube (R928, Hamamatsu) response was corrected using a file supplied by the manufacturer which accounts for its non-linear response. $10 \mathrm{~mm}$ precision quartz cuvettes purchased from Hellma-Analytics were used. In all cases, the optical density of the solutions was below 0.1 . Fluorescence quantum yields were experimentally determined following a previously described method, ${ }^{[40]}$ utilising an integrating sphere (Labsphere, 6 " inner diameter).
The samples were irradiated employing a Xe lamp $\left(\lambda_{\text {exc }}=469 \mathrm{~nm}\right)$ and the signal collected by an optical fibre coupled to Maya2000Pro UV-NIR spectrometer. Lifetime measurements were carried out by using a timecorrelated single-photon-counting instrument (Edinburgh Instruments FLS 920). To excite the samples a picosecond-pulsed EPLED $485(482.0 \mathrm{~nm}$, pulse width FWHM: $100.6 \mathrm{ps}$ ) was used. Deconvolution analysis of the decay kinetics yielded the fluorescence lifetimes. A light-scattering Ludox solution was used to obtain the instrument response function.

Synthesis and characterisation of 2,5-Dibenzyl-3-[4(diphenylphosphoryl)phenyl]-6-phenylpyrrolo[3,4-c]pyrrole-1,4dione (PhODPP). A solution of 2,5-Dibenzyl-3-(4-iodophenyl)-6diphenylpyrrolo[3,4-c]pyrrole-1,4-dione $(0.300 \mathrm{~g}, \quad 0.500 \mathrm{mmol})$, triphenylphosphine $(0.800 \mathrm{~g}, 3.05 \mathrm{mmol})$ and palladium(II) acetate $(0.028$ $\mathrm{g}, 0.125 \mathrm{mmol}, 25 \mathrm{~mol} \%)$ in anhydrous dimethylformamide $(36 \mathrm{~mL})$ was heated to $110-120{ }^{\circ} \mathrm{C}$ and stirred for 18 hours. The solution was concentrated under reduced pressure to give a dark red oil, which was purified by flash column chromatography eluting in dichloromethane/ethyl acetate $(1: 1)$. The product was then suspended in methanol and slowly precipitated overnight. After a second column of the filtered product, eluting in dichloromethane/ethyl acetate (1:1), it was precipitated in dichloromethane/hexane before being dried overnight under vacuum to give a fluorescent orange powder $(0.140 \mathrm{~g}, 42 \%$ yield $) .{ }^{1} \mathrm{H} \mathrm{NMR}\left(\mathrm{CDCl}_{3}\right.$, $400 \mathrm{MHz}) \delta(\mathrm{ppm}): 4.98\left(\mathrm{~s}, 2 \mathrm{H}, \mathrm{CH}_{2}\right), 4.99\left(\mathrm{~s}, 2 \mathrm{H}, \mathrm{CH}_{2}\right), 7.14-7.21(\mathrm{~m}, 4 \mathrm{H}$, ArH), 7.21-7.34 (m, 6H, ArH), 7.42-7.53 (m, 7H, ArH), 7.53-7.61 (m, 2H, $\mathrm{ArH}), 7.63-7.80(\mathrm{~m}, 8 \mathrm{H}, \mathrm{ArH}), 7.81-7.87(\mathrm{~m}, 2 \mathrm{H}, \mathrm{ArH}) .{ }^{13} \mathrm{C} \mathrm{NMR}\left(\mathrm{CDCl}_{3}\right.$, $100 \mathrm{MHz}) \delta(\mathrm{ppm}): 45.76\left(\mathrm{CH}_{2}\right), 109.60(\mathrm{C}), 110.63(\mathrm{C}), 126.63(\mathrm{CH})$, $127.47(\mathrm{CH}), 127.61(\mathrm{C}), 128.63\left(\mathrm{CH}, \mathrm{d},{ }^{3} \mathrm{~J} \mathrm{CP}=12.33 \mathrm{~Hz}\right), 128.72(\mathrm{C})$, $128.82(\mathrm{CH}), 128.92(\mathrm{CH}), 129.07(\mathrm{CH}), 131.06\left(\mathrm{C}, \mathrm{d},{ }^{4} \mathrm{~J}_{\mathrm{CP}}=3.08 \mathrm{~Hz}\right)$, $131.21(\mathrm{C}), 131.69(\mathrm{CH}), 132.05\left(\mathrm{CH}, \mathrm{d},{ }^{2} \mathrm{~J} \mathrm{CP}=10.02 \mathrm{~Hz}\right), 132.19(\mathrm{CH}, \mathrm{d}$, $\left.{ }^{4} \mathrm{~J} \mathrm{CP}=2.31 \mathrm{~Hz}\right), 132.41\left(\mathrm{CH}, \mathrm{d},{ }^{2} \mathrm{~J} \mathrm{CP}=10.02 \mathrm{~Hz}\right), 135.81\left(\mathrm{C}, \mathrm{d},{ }^{1} \mathrm{~J}_{\mathrm{CP}}=101.72\right.$ $\mathrm{Hz}), 137.13$ (C), 137.20 (C), 147.15 (C), 150.09 (C), 162.48 (C=O), 162.71 $(\mathrm{C}=\mathrm{O}) .{ }^{31} \mathrm{P}$ NMR $\left(\mathrm{CDCl}_{3}, 162 \mathrm{MHz}\right) \delta(\mathrm{ppm}): 28.34$. IR $(\mathrm{ATR}) / \mathrm{cm}^{-1}: 3057$ $(\mathrm{ArH}), 3026(\mathrm{ArH}), 2962\left(\mathrm{CH}_{2}\right), 1668(\mathrm{C}=\mathrm{O}), 1615(\mathrm{C}=\mathrm{C}), 1590(\mathrm{C}=\mathrm{C})$, $1494(\mathrm{C}=\mathrm{C}), 1452(\mathrm{C}=\mathrm{C}), 1379\left(\mathrm{CH}_{2}\right), 1345\left(\mathrm{CH}_{2}\right), 1184(\mathrm{P}=\mathrm{O}), 1115(\mathrm{C}-$ H), 717 (ArH), 690 (ArH). Anal. Calculated for $\mathrm{C}_{44} \mathrm{H}_{33} \mathrm{~N}_{2} \mathrm{O}_{3} \mathrm{P}: \mathrm{C}(79.03), \mathrm{H}$ (4.97), N (4.19). Found: C (78.06), H (4.99), N (4.17). Melting point: 196$198^{\circ} \mathrm{C}$.

Synthesis and characterisation of 2,5-Dibenzyl-3-[4(diphenylphosphanyl)phenyl]-6-phenylpyrrolo[3,4-c]pyrrole-1,4dione (PhDPP). A solution of 2,5-Dibenzyl-3-(4-iodophenyl)-6diphenylpyrrolo[3,4-c]pyrrole-1,4-dione (3.15 g, $5.30 \mathrm{mmol})$, triphenylphosphine $(8.37 \mathrm{~g}, 31.9 \mathrm{mmol})$ and palladium(II) acetate $(0.305 \mathrm{~g}$, $1.35 \mathrm{mmol}, 25 \mathrm{~mol} \%)$ in anhydrous dimethylformamide $(380 \mathrm{~mL})$ was heated to $110-120{ }^{\circ} \mathrm{C}$ and stirred for 18 hours. The solution was concentrated under reduced pressure to give a dark red oil, which was purified by flash column chromatography eluting in dichloromethane/ethyl acetate $(9: 1)$. The product was then further purified by a second flash column, eluting in hexane/dichloromethane (1:2), before being concentrated and dried overnight under vacuum to give a fine orange powder $\left(0.450 \mathrm{~g}, 13 \%\right.$ yield). ${ }^{1} \mathrm{H}$ NMR $\left(\mathrm{CDCl}_{3}, 400 \mathrm{MHz}\right) \delta(\mathrm{ppm}): 5.00$ (s, $\left.2 \mathrm{H}, \mathrm{CH}_{2}\right), 5.01\left(\mathrm{~s}, 2 \mathrm{H}, \mathrm{CH}_{2}\right), 7.16-7.22(\mathrm{~m}, 4 \mathrm{H}, \mathrm{ArH}), 7.22-7.40(\mathrm{~m}, 17 \mathrm{H}$, $\mathrm{ArH})$, 7.42-7.52 (m, 4H, ArH), 7.72-7.80 (m, 4H, ArH). ${ }^{13} \mathrm{C} \mathrm{NMR}\left(\mathrm{CDCl}_{3}\right.$, $100 \mathrm{MHz}) \delta(\mathrm{ppm}): 45.60\left(\mathrm{CH}_{2}\right), 109.68(\mathrm{C}), 109.84(\mathrm{C}), 126.65(\mathrm{CH}, \mathrm{d}$, $\left.{ }^{3} \mathrm{~J}_{\mathrm{CP}}=7.71 \mathrm{~Hz}\right), 126.69\left(\mathrm{C}, \mathrm{d},{ }^{4} \mathrm{~J}_{\mathrm{CP}}=6.94 \mathrm{~Hz}\right), 127.35,127.68,127.81$, $128.67\left(\mathrm{CH}, \mathrm{d},{ }^{4} \mathrm{~J}_{\mathrm{CP}}=6.94 \mathrm{~Hz}\right), 128.76,128.85,129.04,129.14,131.42$, $133.47\left(\mathrm{CH}, \mathrm{d},{ }^{2} \mathrm{~J}_{\mathrm{CP}}=19.27 \mathrm{~Hz}\right), 134.00\left(\mathrm{CH}, \mathrm{d},{ }^{2} \mathrm{~J}_{\mathrm{CP}}=20.04 \mathrm{~Hz}\right), 136.04(\mathrm{C}$, d, $\left.{ }^{1} \mathrm{~J}_{\mathrm{CP}}=10.79 \mathrm{~Hz}\right), 137.35\left(\mathrm{C}, \mathrm{d},{ }^{1} \mathrm{~J}_{\mathrm{CP}}=10.02 \mathrm{~Hz}\right), 142.36,142.50,148.41$, 149.07, $162.67(\mathrm{C}=\mathrm{O}), 162.72(\mathrm{C}=0) .{ }^{31} \mathrm{P} \mathrm{NMR}\left(\mathrm{CDCl}_{3}, 162 \mathrm{MHz}\right) \delta(\mathrm{ppm})$ : -4.37. IR (ATR)/ $/ \mathrm{cm}^{-1}$ : 3057 (ArH), 3029 (ArH), $2921\left(\mathrm{CH}_{2}\right), 1677(\mathrm{C}=\mathrm{O})$, $1608(\mathrm{C}=\mathrm{C}), 1591(\mathrm{C}=\mathrm{C}), 1495(\mathrm{C}=\mathrm{C}), 1422(\mathrm{C}=\mathrm{C}), 1384\left(\mathrm{CH}_{2}\right), 1358$ $\left(\mathrm{CH}_{2}\right), 1121(\mathrm{C}-\mathrm{H}), 722(\mathrm{ArH}), 692(\mathrm{ArH})$. Anal. Calculated for $\mathrm{C}_{44} \mathrm{H}_{33} \mathrm{~N}_{2} \mathrm{O}_{2} \mathrm{P}$ : C (80.96), $\mathrm{H}(5.10), \mathrm{N}(4.29)$. Found: C (79.66), $\mathrm{H}(5.04), \mathrm{N}$ (4.13). Melting point: $229-232{ }^{\circ} \mathrm{C}$.

Computational details. The ground state geometries of PhDPP and PhODPP were optimised by means of the density functional M06-2X ${ }^{[41]}$ at 
the $6-31 \mathrm{G}(\mathrm{d})$ level as implemented in Spartan 10 package. ${ }^{[42]}$ In both cases, energy optimised geometries were confirmed by IR analysis, which were characterised by the absence of any imaginary modes, thus consistent with real equilibrium minima. ${ }^{[43-45]}$ The cartesian coordinates for the optimised geometries of both probes can be found in the associated supporting information (SI.4 ESI†).

Cell culture and in-vitro imaging. Stock solutions of both PhDPP and PhODPP were prepared in DMSO for all cell-based experiments, which were used in all cases at DMSO concentrations lower than $0.5 \%(\mathrm{v} / \mathrm{v})$. Cell culture was performed utilising human MCF7 breast cancer cell line, maintained in high glucose Dulbecco's Modified Eagle's Medium (DMEM) supplemented with $10 \%$ foetal bovine serum (FBS), 1\% L-glutamine (200 $\mathrm{mM}$ ) and $1 \%$ antibiotic solution (100 U penicillin/ $0.1 \mathrm{mg} / \mathrm{L}$ streptomycin, all purchased from Sigma-Aldrich. Cells were sub-cultured in appropriate plates/dishes at $15,625 \mathrm{cells} / \mathrm{cm}^{2}$ in phenol red free DMEM without antibiotics and were allowed to rest for $24 \mathrm{~h}$ before being used for experiments. In all cases, cells were incubated at $37{ }^{\circ} \mathrm{C}$ and $5 \% \mathrm{CO}_{2}$ humidified atmosphere. Flow cytometry data was acquired to quantify the cell uptake of PhDPP, PhODPP as well as commercially available staining agent, Mitotracker Red CMXRos (Life Technologies). Cells were subcultured in 6-well plates and treated with different probes (vide infra), thoroughly washed with warm phosphate buffer saline (PBS), detached using trypsin-EDTA (Sigma Aldrich) and resuspended in $500 \mu \mathrm{L}$ of $4 \%$ paraformaldehyde in sterile PBS. Quantitative measurements of DPP uptake were assessed following cell treatment with PhDPP (1.0 and 5.0 $\mu \mathrm{g} / \mathrm{mL}$ ) and PhODPP $(0.5,1.0$ and $5.0 \mu \mathrm{g} / \mathrm{mL}$ ), which were incubated for 1h. Cytometry data of DPP uptake was detected in a FC500 (Beckman Coulter, $\lambda_{\mathrm{exc}}=488 \mathrm{~nm}$ and $\lambda_{\mathrm{em}}=514-542 \mathrm{~nm}$ ). The quantification of mitochondrial accumulation of PhODPP and commercially available Mitotracker Red CMXRos was performed following cell treatment with 250 $\mathrm{nM}$ of each probe, at $10 \mathrm{~min}$ and $1 \mathrm{~h}$ incubation times, as well as simultaneous treatment with $250 \mathrm{nM}$ of both probes (1h incubation) Cytometry data for PhODPP and Mitotracker Red CMXRos was acquired in an Astrios cell sorter (Beckman Coulter, $\lambda_{\mathrm{exc}}=488 \mathrm{~nm}$ and $\lambda_{\mathrm{em}}=500$ $526 \mathrm{~nm}$ for PhODPP and $\lambda_{\mathrm{exc}}=561 \mathrm{~nm}$ and $\lambda_{\mathrm{em}}=604-624 \mathrm{~nm}$ for Mitotracker Red CMXRos). Acquired flow cytometry data was then analysed using Kaluza software provided by the manufacturer. The median fluorescence intensity values of homogeneous and healthy cell populations (\% Gated) was used to quantify the cell uptake. Confocal microscopy analyses were carried out in cells that were sub-cultured in glass dishes (CELLview ${ }^{\mathrm{TM}}$ culture dish, Greiner Bio-One, Germany), that received the appropriate treatment (vide infra) and that were incubated with Hoechst 33342 at $5 \mu \mathrm{g} / \mathrm{mL}$ for $10 \mathrm{~min}$ in all cases. Cells were then thoroughly washed with warm PBS and preserved with $4 \%$ paraformaldehyde in sterile PBS. The visualisation of intracellular probe accumulation was performed in a TCS SPE Leica confocal microscope using a 40x objective. Obtained images were then analysed using Image $J$ software (Rasband). To characterise the cell uptake, cells were incubated with $1 \mu \mathrm{g} / \mathrm{mL}$ PhDPP/PhODPP in DMSO and $250 \mathrm{nM}$ Mitotracker Red CMXRos for $1 \mathrm{~h}$. To further characterise the PhODPP mitochondrial accumulation, the cells were treated with $250 \mathrm{nM}$ of PhODPP and Mitotracker Red CMXRos for $1 \mathrm{~h}$. The cell viability of PhDPP and PhODPP was determined by the resazurin based metabolic assay PrestBlue ${ }^{T M}$ (Thermofisher Scientific) at three different passage numbers. After 24h of cell sub-culture in black 96 -well plates (Nunclon ${ }^{\mathrm{TM}}$ Delta surface, Thermo Scientific), cells were treated with serial dilutions of PhDPP and PhODPP in DMSO 0.17-21.38 $\mu \mathrm{g} / \mathrm{mL}(32 \mu \mathrm{M}-250 \mathrm{nM}$ ), vehicle control (DMSO 0.02$2 \% \mathrm{v} / \mathrm{v}$ ), positive control (TritonX $1 \%$ ) and negative control (cell culture media). Cells were incubated for $1 \mathrm{~h}$ after treatment, then washed with warm PBS three times and incubated for $1 \mathrm{~h}$ with prestoBlue ${ }^{\mathrm{TM}}$ in cell culture media (ratio 1:9 (v/v), $100 \mu \mathrm{L} /$ well). Lastly, the luminescence $\left(\lambda_{\text {exc }}\right.$ $=560 \mathrm{~nm}$ and $\lambda_{\mathrm{em}}=600 \mathrm{~nm}$ ) was subsequently detected in a TECAN microplate reader (Tecan Trading AG, Switzerland).

\section{Acknowledgements}

The authors thank the lab of Dr Uwe Pischel (University of Huelva, Spain) for the determination of the fluorescence lifetimes presented in this manuscript. CJM, JC-C and SML acknowledge funding from the Brazilian Conselho Nacional de Desenvolvimento Cientifico e Tecnologico, CNPq [422329/20185]. CA and TFA acknowledge funding from the Engineering and Physical Sciences Research Council [EP/N03371X/1].

Keywords: Diketopyrrolopyrroles • Mitochondria staining • Neutral probe $\cdot$ Nanomolar

[1] M. Abo, Y. Urano, K. Hanaoka, T. Terai, T. Komatsu, T. Nagano, J. Am. Chem. Soc. 2011, 133, 10629-10637.

[2] E. W. Miller, A. E. Albers, A. Pralle, E. Y. Isacoff, C. J. Chang, J. Am. Chem. Soc. 2005, 127, 16652-16659.

[3] B. C. Dickinson, C. Huynh, C. J. Chang, J. Am. Chem. Soc. 2010 132, 5906-5915.

[4] K. J. Bruemmer, R. R. Walvoord, T. F. Brewer, G. Burgos-Barragan, N. Wit, L. B. Pontel, K. J. Patel, C. J. Chang, J. Am. Chem. Soc. 2017 139, 5338-5350.

[5] B. C. Dickinson, C. J. Chang, J. Am. Chem. Soc. 2008, 130, 96389639 .

[6] Roopa, N. Kumar, V. Bhalla, M. Kumar, Chem. Commun. 2015, 51, 15614-15628.

[7] A. T. Hoye, J. E. Davoren, P. Wipf, M. P. Fink, V. E. Kagan, Acc. Chem. Res. 2008, 41, 87-97.

[8] H. Zhu, J. Fan, J. Du, X. Peng, Acc. Chem. Res. 2016, 49, 21152126.

[9] K. Henze, W. Martin, Nature 2003, 426, 127-128.

[10] P. I. Moreira, X. Zhu, X. Wang, H. -g. Lee, A. Nunomura, R. B. Petersen, G. Perry, M. A. Smith, Biochim. Biophys. Acta - Mol. Basis Dis. 2010, 1802, 212-220.

[11] C. Zhang, L. Long, C. Shi, Adv. Ther. 2018, 1, 1800069.

[12] J. R. Lakowicz, Principles of Fluorescence Spectroscopy, Springer, 2006.

[13] H. N. Kim, W. X. Ren, J. S. Kim, J. Yoon, Chem. Soc. Rev. 2012, 41, 3210-3244.

[14] W. Kühlbrandt, BMC Biol. 2015, 13, DOI 10.1186/s12915-015-0201-x.

[15] G. A. Perkins, T. G. Frey, Micron 2000, 31, 97-111.

[16] S. Hoppins, L. Lackner, J. Nunnari, The Machines That Divide and Fuse Mitochondria, 2007.

[17] O. Schmidt, N. Pfanner, C. Meisinger, Nat. Rev. Mol. Cell Biol. 2010 11, 655-667.

[18] P. Gao, W. Pan, N. Li, B. Tang, Chem. Sci. 2019, 10, 6035-6071.

[19] Y. Chen, C. Zhu, J. Cen, Y. Bai, W. He, Z. Guo, Chem. Sci. 2015, 6 3187-3194.

[20] J. Lv, Y. Chen, F. Wang, T. Wei, Z. Zhang, J. Qiang, X. Chen, Dyes Pigments 2018, 148, 353-358.

[21] R.-R. Ye, C.-P. Tan, L.-N. Ji, Z.-W. Mao, Dalton Trans. 2016, 45, 13042-13051.

[22] X. Zhang, Q. Ba, Z. Gu, D. Guo, Y. Zhou, Y. Xu, H. Wang, D. Ye, H. Liu, Chem. - Eur. J. 2015, 21, 17415-17421.

[23] L. V. Johnson, M. L. Walsh, L. B. Chen, Proc. Natt. Acad. Sci. U. S. A 1980, 77, 990-994.

[24] L. F. Mottram, S. Forbes, B. D. Ackley, B. R. Peterson, Beilstein J. Org. Chem. 2012, 8, 2156-2165.

[25] T. Gayathri, S. Karnewar, S. Kotamraju, S. P. Singh, ACS Med. Chem. Lett. 2018, 9, 618-622.

[26] H. M. Smith, High Performance Pigments, Wiley-VCH, 2002.

[27] O. Wallquist, R. Lenz, Macromol. Symp. 2002, 187, 617-629.

[28] Z. M. Hao, A. lqbal, Chem. Soc. Rev. 1997, 26, 203-213.

[29] R. M. Christie, Colour Chemistry, The Royal Society Of Chemistry, 2001.

[30] W. Herbst, K. Hunger, Industrial Organic Pigments, Wiley-VCH, 2004

[31] M. Grzybowski, E. Glodkowska-Mrowka, V. Hugues, W. Brutkowski M. Blanchard-Desce, D. T. Gryko, Chem. - Eur. J. 2015, 21, 91019110.

[32] J. Wang, L. Liu, W. Xu, Z. Yang, Y. Yan, X. Xie, Y. Wang, T. Yi, C. Wang, J. Hua, Anal. Chem. 2019, 91, 5786-5793.

[33] X. Xie, J. Wang, Y. Yan, X. Zhang, C. Liu, J. Yang, J. Hua, Analyst 2018, 143, 5736-5743.

[34] C. Du, S. Fu, X. Ren, X. Wang, Z. Wang, J. Zhou, H. Wang, New J. Chem. 2018, 42, 3493-3502.

[35] J. Wang, Y. Hang, H. Tan, T. Jiang, X. Qu, J. Hua, J. Photochem. Photobiol. Chem. 2017, 346, 265-272.

[36] F. Y. Kwong, K. S. Chan, Chem. Commun. 2000, 1069-1070. 
[37] J. Calvo-Castro, M. Warzecha, I. D. H. Oswald, A. R. Kennedy, G. Morris, A J. McLean, C. J. McHugh, Cryst. Growth Des. 2016, 16 1531-1542.

[38] M. Warzecha, J. Calvo-Castro, A. R. Kennedy, A. N. Macpherson, K. Shankland, N. Shankland, A. J. McLean, C. J. McHugh, Chem. Commun. 2015, 51, 1143-1146.

[39] M. Kaur, D. H. Choi, Chem. Soc. Rev. 2015, 44, 58-77.

[40] J. C. De Mello, H. F. Wittmann, R. H. Friend, Adv. Mater. 1997, 9, 230-232.

[41] Y. Zhao, D. G. Truhlar, Theor. Chem. Acc. 2008, 120, 215-241.
[42] Y. Shao, L. F. Molnar, Y. Jung, J. Kussmann, C. Ochsenfeld, S. T. Brown, A. T. B. Gilbert, L. V. Slipchenko, S. V. Levchenko, D. P. O'Neill, et al., Phys. Chem. Chem. Phys. 2006, 8, 3172-3191.

[43] A. Szabo, N. S. Ostlund, Modern Quantum Chemistry: Introduction to Advanced Electronic Structure Theory, McGraw-Hill, 1989.

[44] F. Jensen, Introduction to Computational Chemistry, John Wiley And Sons, 2007.

[45] A. R. Leach, Molecular Modelling. Principles and Applications, Prentice-Hall, 2001. 


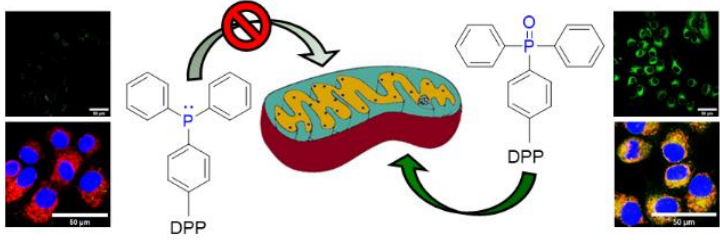

Selective mitochondrial imaging by a neutral probe at the nanomolar level is demonstrated employing a rationally designed triphenylphosphine oxide-bearing diketopyrrolopyrrole.
Thais F. Abelha, Graeme Morris, Sandro M. Lima, Luis H. C. Andrade, Andrew J. McLean, Cameron Alexander, Jesus Calvo-Castro* and Callum J. McHugh *

Page No. - Page No.

Development of a neutral diketopyrrolopyrrole phosphine oxide for the selective bioimaging of mitochondria at the nanomolar level 